\title{
An Intelligent, Multi-Transducer Signal Conditioning Design for Manufacturing Applications
}

I. Sillitoe (corresponding author),

I.Sillitoe@wlv.ac.uk

School of Engineering

University of Wolverhampton

Telford, Shropshire, TF2 9NT, United Kingdom.

M.Button and E. Owhonda

Phoenix Calibration \& Services Limited

Brierley Hill, West Midlands, DY5 1QA,United Kingdom.

martin.button@phoenixcalibration.co.uk

edison@phoenixcalibration.co.uk

\begin{abstract}
This paper describes a flexible, intelligent, high bandwidth, signal conditioning reference design and implementation, which is suitable for a wide range of force and displacement transducers in manufacturing applications. The flexibility inherent in the design has allowed more than 10 specialised transducer conditioning boards to be replaced by this single design, in a range of bespoke mechanical test equipment manufactured by the authors. The board is able to automatically reconfigure itself for a wide range of transducers and calibrate and balance the transducer. The range of transducers includes LVDT, AC/DC strain gauge and inductive bridges, and a range of standard industrial voltage current interface transducers. Further, with a minor lowcost addition to the transducer connector, the board is able to recognise the type of transducer, reconfigure itself and store the calibration data within the transducer, thereafter allowing a plugand-play operation as transducers are changed. The paper provides an example of the operation in typical manufacturing test application and illustrates the stability and noise performance of the design.
\end{abstract}

Keywords: automatic signal conditioning, multi-transducer, manufacturing test applications

\section{INTRODUCTION}

The advantages of smart or intelligent sensors have long been recognised in a wide range of applications (e.g. [1],[2],[3],[5],[6],[7]). Amongst the potential advantages, intelligent sensors can provide measurements with higher signal to noise ratios (since the signal conditioning can be located close to or within the transducer) and they can be made to automatically adapt to a particular transducer's characteristics, allowing balancing or calibration to take place at the transducer. In doing so, intelligent sensors can reduce the complexity and cost of the overall system (see [3] for an extensive list of their perceived advantages). 
However, the majority of the intelligent sensors include signal conditioning circuitry which is specific to a single type of transducer. Also with the notable exception of the approach taken in [4], far less attention has been paid to the design of flexible conditioning circuitry which could interface to a wide range of industrial transducers.

This paper describes a single reference design and board intended to address the practical needs of flexible signal conditioning for a wide range of transducers common to industrial measurement applications.

The structure of the paper is as follows. Section 2 briefly describes the operating requirements of two applications, in order to illustrate the flexibility of the design in a manufacturing context. Section 3 provides an overview of the principal features of the design. Section 4 then describes the structure of the design in detail, with respect to the applications given in section 2. The paper concludes with a brief overview of the application of the design in practice.

\section{Application Areas}

Phoenix manufacture bespoke test equipment to evaluate the mechanical properties of a wide range of products and materials. The following test applications are described to illustrate the requirements made of the design.

One of the most typical cases for moderately fast Phoenix test systems are compaction simulators used in the pharmaceutical industry (see Figure 1). Compaction simulators evaluate the effect of different force/displacement profiles, on the compaction of powders in the production of tablets. These generally have maximum actuator velocities between $1.5-3 \mathrm{~m} / \mathrm{s}$ and a data acquisition period being no less than $100 \mathrm{~ms}$ in duration. The test performed by these machines typically consists of a force/displacement profile made of 4096 points independently of the test's duration.

For a test duration of $100 \mathrm{~ms}$, it would follow that the equivalent data acquisition rate would be close to $40 \mathrm{kHz}$. However, the limited bandwidth of the loading frame restricts the useful bandwidth of the signal to $20 \mathrm{kHz}$. Simple compaction simulators use strain gauge load cells for force measurement and linear variable differential transformers (LVDTs) for the displacement measurement, using a typical excitation frequency of $5 \mathrm{kHz}$ and a 2-pole anti-aliasing filter with a roll-off at $250 \mathrm{~Hz}$. In order that the load measurement channel should have a similar frequency response to that of the displacement, a matching filter is often fitted to the load amplifiers. In such a case there is a considerable loss of high frequency detail in the acquired signal. The compaction simulators developed by Phoenix improve upon this by using magnetostrictive or inductive displacement sensors which have a bandwidth of $10 \mathrm{kHz}$. Because the sensor bandwidth is higher, the displacement filtering does not need to be so severe, and so the filtering of the load channel can be reduced to match. This enables the fine detail to be retained in the acquired signal. However, whilst strain-gauged load cells are applicable for such tests, they are not the best choice for very high-speed impact test measurements.

The test machines with the most demanding requirements tend to be those used for 'single-shot' tests. These vary widely in their performance requirements, but the essence of such a machine is that it is configured to perform a single high-speed impact test. Typical applications are the testing of material properties during forging, ballistics, and crash conditions in automotive applications. For such tests the emphasis is upon the control of the correct actuator velocity rather than upon the need to follow a sophisticated displacement profile as is the case in a compaction simulator. In common with compaction simulators, single-shot machines require high bandwidth displacement transducers and so magnetostrictive or inductive transducer types are used for the reasons explained above.

However, a single-shot machine differs from the compaction simulator in that the bandwidth of the load measurement must be significantly wider, owing to the impulse nature of the impact. In such cases, conventional strain-gauge cells often fail to meet the bandwidth requirements and also tend to 'ring' when struck (often at frequencies in the hundreds to thousands of hertz). To overcome this limitation, piezoelectric cells with charge amplifiers are often employed to measure the impact forces. Since piezoelectric cells have higher bandwidths, owing to their higher stiffness, they also have a reduced ringing response. The charge-amplifiers used with such cells typically have bandwidths of up to $20 \mathrm{kHz}$, which is sufficient to characterise most impacts. However, unlike strain gauge load cells, piezoelectric 
load cells also exhibit significant static drift and thus the amplifier must be balanced (or 'zeroed') immediately before each test event.

The applications above illustrate the requirements of a flexible signal conditioning design, since for these two applications alone the signal conditioning is required to contend with a range of transducers (in these cases LVDT, magnetostrictive, inductive, strain gauge and piezoelectric with charge amplifier), two different forms of balancing requirement, three different forms of excitation and two bandwidth requirements. The following section provides an overview of the features of the design and, in addition, illustrates how the design fulfils the requirements described above. Section 4 provides a detailed description of the architecture of the design.

\section{Features Of The Design}

Figure 2 illustrates where, in a larger system diagram, the signal conditioning design would operate. It can be seen in Figure 2 that each design can serve up to a maximum of two transducers and that the conditioned analogue signal is available as an output from the board. Thus by combining the board with alternative analogue to digital converters (ADCs) of differing specification, a range of bespoke modules can be created, further increasing the flexibility of the design. In addition, the board has limited built-in self-test and self-diagnosis and can communicate the results over the bus or via a local USB connection to the wider system or to a maintenance engineer.

Figure 1: Compaction simulator (File: Fig 1 compaction simulator.jpg)

Figure 2: Block diagram of typical usage of the design (File: Fig 2 Block diagram.pdf)

The design is able to automatically reconfigure itself for a wide range of transducers and then calibrate and balance the transducer in question. The range of transducers that the design can work with includes (but is not limited to) LVDT [3,4] (both single-sided and complementary excitation), AC/DC strain gauge (including pressure transducers, extensometers) potentiometric displacement transducers (both linear and rotary) and inductive bridges (both full and half bridges). The board can interface to a range of standard industrial voltages (e.g. 0 to 5VDC, 0 to 10VDC, -10 to +10VDC, un-scaled DC outputs) and current-source transducers (both 4 to $20 \mathrm{~mA}$ and 0 to $20 \mathrm{~mA}$ ). To allow the automatic balancing of the transducers which require external excitation (e.g. LVDTs), the design can also be configured to provide a range of excitation/supply voltages for the transducer (e.g. 5 or $10 \mathrm{kHz} 1$ to $5 \mathrm{~V}$ RMS AC single-sided or complementary, 5 or 10V DC single sided or complementary 5V DC supply, 24V DC supply, +/-15V DC supply) for transducers.

The design described here differs from previous designs $[4,8,9,10]$ in that its architecture is based around a single analogue signal path under the control of a microcontroller. In order to achieve the accuracy requirement for such applications, the board uses a novel two stage Vernier-like gain stage within the analogue signal path. This approach provides a higher bandwidth path and lower noise levels than typically found in monolithic switched-capacitor designs. The higher bandwidth admits the possibility of transient measurement (i.e. impact testing), not typically found in previous designs.

The microcontroller can be configured externally, via the bus or USB connection. It can also configure itself automatically based upon the transducer attached. With the minor low-cost addition of a 1-wire EEPROM [13] to the transducer connector, automatic configuration is made possible. This allows the design to identify the transducer and reconfigure itself. It can also store calibration data within the transducer, thereafter allowing a plug-and-play operation as transducers are changed.

\section{DETAILED OPERATION}

The following subsections describe the three major elements of the design namely, the analogue path from transducer to ADC, the excitation element which provides the voltage excitation or reference for various forms of transducer and finally, the function of the microcontroller within the operation of the board. 


\section{1 analog Signal path}

Figure 3: Overall schematic diagram of the analogue path within the reference design

(File: Fig 3 Block diagram.pdf)

Figure 3 provides an overall schematic diagram of the analogue path. The following explanation of Figure 3 follows the analogue path from input to output.

\subsubsection{INPUT CONDITIONING}

The amplifier board is designed to work with either a single sensor $(A)$, or two similar sensors $(A$ and $B)$ and therefore two separate input conditioning channels exist within the design, Stage $A$ and Stage $B$.

Sensor outputs can range from a few millivolts to a few volts DC, a DC current, or in the case of LVDTs signals between $1-15 \mathrm{~V}$ RMS. Each form of signal must be conditioned and finally brought to the required board output range of $+/-10 \mathrm{VDC}$. In order to condition such a wide dynamic range of signals, the input condition block must be able to provide a gain of the order of hundreds, or in other cases the signal may need to be attenuated to a maximum of $4 \mathrm{~dB}$. To achieve the necessary gain, the signal is amplified by an instrumentation amplifier, the approximate gain of which is set by the microcontroller via a digital potentiometer [11]. When attenuation is required, a resistor chain reconfigured by a number of CMOS switches is used to attenuate the signal. The microcontroller uses a combination of the digital potentiometer and CMOS switches to provide the necessary configurable input conditioning.

\subsubsection{SENSOR SELECTION}

The sensor selection stage allows the board to select whether Stage $A$, Stage $B$, the difference of the two signals or their average, are to be processed. The microcontroller controls this selection using $\mathrm{I} / \mathrm{O}$ lines to CMOS switches which alter the configuration of a difference amplifier (sensor selection). Typically the dual sensor application would be found when using extensometry in a tensile testing machine.

\subsubsection{PRE-ZERO}

There are situations when a sensor (in most cases, a resistive bridge sensor) may be initially unbalanced. To correct the imbalance, a digital potentiometer is connected across the bridge to provide compensation. The microcontroller adjusts the potentiometer through the SPI control line (pre-zero potentiometer adjust), in a similar fashion to [12] to achieve a balanced condition. The Pre-Zero is only applied to stage $A$, as it is not required in dual sensor situations where the signals from Stage $A$ and Stage $B$ are either summed, subtracted or averaged.

\subsubsection{Demodulator, Demodulator REFERENCE and ANTI-ALIAS FILTER}

Where the sensor is of an AC type, such as an LVDT, the AC signal must be demodulated before it can be processed further. The demodulation process in Figure 3 uses a phase-sensitive detector with a reference taken from the out-going $A C$ excitation. The principle of operation is shown in Figures 4 and 5 and based upon the selective inversion of alternate half-cycles of the signal. A reference square wave is generated from the excitation waveform (fig. 6), and used to operate a CMOS switch which controls which portions of the signal are to be inverted. While the reference square wave is ' 0 ' the waveform is inverted, while it is ' 1 ' the waveform is not inverted. The resultant rectified waveform is subsequently filtered to obtain a DC measurement of the transducer position.

In practice, there is usually a phase shift $(\theta)$ between the excitation and the signal, as shown in Figures 4 and 5 . The phase of the demodulator is therefore adjusted by the microcontroller so that distortion of the demodulated signal is minimized. The adjustment of the phase is controlled by varying the resistance of a digital potentiometer via the I2C bus (phase reference). When an AC sensor signal has been demodulated there is still a considerable AC component to the signal. This is removed by a low-pass filter which follows the demodulator circuit.

Figure 4: Poorly adjusted demodulation (File: poor demod.pdf) 
Figure 5: Correctly adjusted demodulation (File: correct demod.pdf)

\subsubsection{Fine Gain and Coarse Gain Stages}

Although the configurable input stages include the possibility for both gain and attenuation of the signal, the signal is still not scaled at this point. The output of the board must be accurately scaled and calibrated to the standard board output voltage of $+/-10 \mathrm{~V} D C$ full scale. The coarse gain is implemented using a digital potentiometer in the feedback loop of an operational amplifier. This gives a fairly 'steppy' and nonlinear control of gain. To allow precise correction of the gain, a multiplying digital to analogue converter (DAC) is used in the fine gain stage. The microcontroller controls the coarse gain digital potentiometer and multiplying DAC via the SPI lines Range Potentiometer Control and Range MDAC Control respectively.

\subsubsection{AUto-ZERo (DATUM OfFSET)}

Automatic datum offsetting ('taring', or removal of initial measured value) is achieved by using a DAC generated analogue signal, summed into the signal path, prior to the fine and coarse gain stages. Typically, this signal is sourced off-board, but a DAC is provided on-board for those situations where a self-contained system is required. A CMOS switch is used by the microcontroller to select either on-board or off-board taring source.

\subsubsection{OUtPUt DRIVERS AND OVERLOAD DETECTION}

The signal conditioning board has two buffered outputs; one for the control system's ADC, the other being a monitoring output available for general purpose use.

In some applications, such as when the board is configured as a load amplifier, it is also necessary to know when the board output is out of range, to avoid damaging the load cell. The overload circuit incorporates a 'window' comparator whose output is active when the board output exceeds the specified level, in either direction. The logical sense of the overload output can be set using jumpers, to either normal or inverse. The overload signal is available to the wider system as a safety output signal. In addition, the microcontroller also monitors the overload status of the sensor using an $\mathrm{I} / \mathrm{O}$ pin, (uC Overload Monitoring).

\subsection{SENSOR EXCITATION}

Figure 6: Excitation circuitry (File: Excitation circuitry.pdf)

The principle of operation of the amplifier board is to rely on the precisely controlled sensor excitation so that the transducer sensor signal can then be regarded as an absolute representation of the measured value. To achieve this, a choice of precision $A C$ and $D C$ references is required. These are provided by the board, although it is also possible to source these off-board, through the edge-connector. Depending on the application, the board can be configured to use either AC or DC excitation. The microcontroller controls this selection using a CMOS switch controlled by the AC DC Select control line as shown in the block diagram. Once either AC or DC has been selected, further settings are made as described below:

\subsubsection{REFERENCE}

The $\mathrm{DC}$ reference section provides either precision $\mathrm{DC}+5 \mathrm{~V}$ or $+10 \mathrm{~V}$ as required. This can be selected by the microcontroller using a CMOS switch through the DC Ref Select I/O line.

\subsubsection{MASTER OSCILLATOR}

The master oscillator uses a Wein Bridge oscillator to generate a highly stable low-distortion sine-wave reference for use with $\mathrm{AC}$ sensor types. The frequency and amplitude of the oscillator is controlled via the I2C bus from the microcontroller using the Oscillator frequency and Amplitude control lines.

\subsubsection{EXCITATION BUFFER}

The master oscillator and DC references source only a limited amount of current. Therefore, in order to use these signals to excite sensors, such as LVDTs or strain-gauge bridges, the excitation is buffered. The buffer not only provides sufficient current for excitation of the sensor, but it can also be configured to provide single-sided or complementary outputs to suit other forms of sensor and to increase the commonmode rejection of the system. 


\subsubsection{EXCITATION TYPE}

For sensors which do not require $A C$ or $D C$ references but require power supplies (which are then internally regulated by the sensor) the Excitation Type circuit can switch between the various power rails within the conditioning board to provide the necessary output. This accommodates sensors which require non precision excitations such as $15 \mathrm{~V}$ unipolar, bipolar and $24 \mathrm{~V}$, which can be routed from the power supply rails, without the need for buffering. The selection of the particular excitation is achieved by a network of signal relays controlled by the microcontroller I/O pins through MOSFET drivers by the Excitation Type Select control lines.

\subsubsection{BOARD SELECTION}

A selection system, which is under control of the overall system firmware, can select or de-select the board. In the de-selected state the sensor inputs and outputs are galvanically isolated using relays.

\subsection{MICROCONTROLLER}

Figure 7: The principal interfaces of the microcontroller (File: micro interfaces.pdf)

The microcontroller lies at the heart of the board's re-configurability and it not only controls the analogue signal path through the board but also allows the board to communicate with the host control system via data lines on the board's edge connector. Information from the wider control system can also be interpreted by the microcontroller and used to determine the states and parameters with which the board will operate. During the initialization phase, the board can also identify itself to the control system by supplying an identification code (IDENT) through the bus. The IDENT informs the control system of the type of transducer the board is currently configured for, and the available working ranges.

\subsubsection{One Wire Identification}

With a minor low-cost addition to the transducer's connector, the microcontroller is able to uniquely identify a particular transducer connected to the board. This allows the board to automatically select the pertinent configurations of the analogue signal path and excitation stage to suit that particular transducer, while preventing configurations that could potentially damage the transducer. Once the transducer had been calibrated, calibration data and other settings specific to that particular sensor can be stored within EEPROM located in the transducer's connector. This provides the board with a flexible 'plug and play' operation which is necessary if the design is to efficiently configure itself for a wide range of transducers. The communication between transducer connector and the board is based upon the 1-wire sensor bus [10] and requires only the addition of a single low-cost chip to provide both the identification and EEPROM.

Figure 8: An illustration of the one wire identification implementation (File: Fig 8 one wire.jpg)

\section{TYPICAL APPLICATION}

A typical testing machine application often has three sources of measurement namely stroke, extension and load. These correspond to the measurement of the position of the testing machine's hydraulic actuator, the deformation of test specimen measured by an accurate extensometer, and the measurement of the force applied to the test specimen, often measured by a strain-gauged load cell. The type of stroke and load sensor are fixed during design of the testing machine, but the type and form of extensometer can vary with customer requirements, and may be changed to suit a particular test regime by the customer at any time. The form of extensometer transducer may be of single or dual LVDT type, or DC bridge. An example of such a system used for compression testing of a steel sample is shown in figure 9. Three boards were used to control the machine, where each board automatically configured itself for its task using one-wire identification mechanism describe in section 4 . The control system containing the boards is shown to the right of the figure. Figure 10 shows the board itself.

Figure 9. Picture of overall machine during test (File: Fig 9 Application Machine.jpg) Figure 10. The board used in a control system (File: Fig 10 Board.jpg) 
In another recent application, it was required that a DC bridge extensometer, with a working range of +/$5 \mathrm{~mm}$, should produce measurements accurate to the order of micrometres over its full working range. This was accomplished in the new design, by creating two measurement profiles within the board, one coarse, with a range of $+/-5 / 2 / 1 / 0.5 \mathrm{~mm}$ and the other fine, with a range of $+/-0.5 / 0.2 / 0.1 / 0.05$ $\mathrm{mm}$. These profiles were calibrated automatically by the board. By programming the conditioning board to alter the break point of the anti-aliasing filter from $250 \mathrm{~Hz}$ to $25 \mathrm{~Hz}$, it was then possible to control the hydraulic actuator of the testing machine, to within $7 \mu \mathrm{m}$. Using conventional signal conditioning designs, this process would have required two separate signal conditioning boards, one for each range profile, manual modification of the filter break-point and manual calibration.

\subsection{Stability and Noise Performance}

The test machine performed $1 \mathrm{~Hz}$ sine-wave loading cycle in load control for a period of 48 hours. The stability and noise performance of the board was determined by monitoring the load cell output and comparing it to an additional manually calibrated proving load cell in the loading chain. The output of the calibration cell and displacement data were acquired for comparative purposes. The ambient temperature of the system ( shown in figure 11) was left uncontrolled during the period of the test.

Figure 11. Variation of temperature during the test. (File: Temp_Trajectory.pdf)

For the sake of clarity, the plots shown in figures 12, 13 and 14 (illustrating the stoke trajectory, force measurements of the manually calibrated proving load cell and the load cell controlled by the new design), indicate only the extrema of the cycles, rather than the individual samples taken over the entire loading cycle.

Figure 12 The extrema of the stroke trajectory during the test. (File: Stroke_Trajectory.pdf) Figure 13 The extrema of the manually calibrated proving load cell during the test. (File:

\section{Proving_Loadcell_Trajectory.pdf)}

Figure 14 The extrema of the load cell automatically configured and calibrated by the new design. (File: Automatic_Loadcell_Trajectory.pdf)

A detailed analysis of the data, illustrated by figures 13 and 14 together with the results of additional bench tests over a wider temperature range, indicate that the new design is not only able to automatically configure and balance the load cell, but that the thermal drift of the new approach is a half that of the previous design and that the signal to noise ratio is improved by $\sim 3 \mathrm{~dB}$.

\section{SUMMARY}

This paper has presented a reference design and implementation of a flexible signal conditioning system which is able to automatically identify, calibrate/balance and process a very wide range of transducer and sensor signals commonly used within industrial applications. The design has currently replaced 10 separate single function products, providing reductions in manufacturing, maintenance and design costs. The original products relied upon manual calibration and sensor balancing, through the use of trim potentiometers. The new design is able configure and balance itself automatically and has improved upon the thermal drift (by a factor of 2 ) and signal-to-noise ratio characteristics $(\sim 3 \mathrm{~dB})$ of the original products. This has been achieved through the use of a two-stage gain control and automatic balancing and demodulation techniques. When these approaches are used in tandem they provide a more versatile control of the gain than is possible with purely passive devices and can be used to compensate for their thermal drift.

The flexibility of the design is derived from the wide variations possible in the analogue signal path, excitation circuitry and the 'plug and play' nature of the transducer interface. In addition, given the modularity of the design, the characteristics of a particular implementation of the design can be further tailored for a bespoke application (e.g. in terms of signal bandwidth, signal to noise ratio or cost) by the choice of components used to implement the system blocks, without modification to the architecture of the design or the printed circuit board layout. 
Further work will include an expansion of the self-test and diagnostic features of the board, in particular the ability of the board to generate self-test stimuli during system boot, and real-time remote monitoring during operation.

\section{ACKNOWLEDGEMENTS}

This work was undertaken as part of the Innovate UK, Technology Strategy Board, Knowledge Transfer Partnership Grant No. 8791.

\section{REFERENCES}

[1] S. Pal and A. Rakshit: "Development of Network Capable Smart Transducer Interface for Traditional Sensors and Actuators", Sensor and Actuators A, vol.112, pp. 381-387, 2004.

[2] J. G. Rocha, C. Couto and J. H. Correia: "Smart Load Cells: An Industrial Application", Sensors and Actuators A: Physical, vol(85), No 1-3, pp. 262-266, 2000.

[3] G. Smith and M. Bowen: "Considerations for the utilization of smart sensors", Sensors and Actuators A: Physical, vol(47), No 1-3, pp. 521-524, , 1995.

[4] J. Zhang, J. Zhou, and A. Mason: "Highly Adaptive Transducer Interface Circuit for Multiparameter Microsystems", IEEE Transactions on Circuits and Systems I: Regular Papers, 54(1), pp. 167-178, 2007.

[5] E. D. Kyriakis-Bitzaros, N. A. Stathopoulos, S. Pavlos, D. Goustouridis and S. Chatzandroulis, "A Reconfigurable Multichannel Capacitive Sensor Array Interface", IEEE Transactions on Instrumentation and Measurement, vol. 60, pp. 3214-3221, 2011.

[6] Y. Huang, T. Tzeng, T. Lin, C. Huang, P. Yen, P. Kuo, et al., "A Self-Powered CMOS Reconfigurable MultiSensor SoC for Biomedical Applications", Solid-State Circuits IEEE Journal of, vol. 49, pp. 851-866, 2014.

[7] S. Kim, J. Kim, J. Choi and W. Eom, "Dual-mode wide-range linear CMOS interface circuit for resistive sensors", Electronics Letters, vol. 50, pp. 1575, 2014.

[8] A. Drumea, A. Vasile, M. Comes, and M. Blejan: "System on Chip Signal Conditioner for LVDT Sensors", The Proceedings of the First Electronics System integration Technology Conference, pp. 629-634, 2006.

[9] R.S, Weissbach, D.R. Loker, and R.M. Ford, "Test and comparison of LVDT signal conditioner performance", Proceedings of the 17th IEEE Instrumentation and Measurement Technology Conference (IMTC 2000), vol.2, pp. $1143-1146,2000$

[10] P.-M Nicolae, D. Stanescu, M. Nicolae, and I.-R. Bojoi: "Considerations on Signals Conditioning Circuits", 11th International Conference on Electrical Power Quality and Utilisation (EPQU), pp. 1-6, 2011.

[11] B. Sevcik: "Modeling and Signal Integrity Testing of Digital Potentiometers", Proceedings of the 17th International Conference Mixed Design of Integrated Circuits and Systems (MIXDES), pp. 570-575, 2010.

[12] M. Leinonen, J. Juuti, J. and H. Jantunen: "Interface Circuit for Resistive Sensors Utilizing Digital Potentiometers", Sensors and Actuators A: Physical, vol(138), No 1, pp. 97-104, 2007.

[13] "Book of iButton Standards", Maxim/Dallas Semiconductor, Chapter 1, pp. 9, edition 08-12-97 http://pdfserv.maximintegrated.com/en/an/AN937.pdf (Visited 25/01/15) 


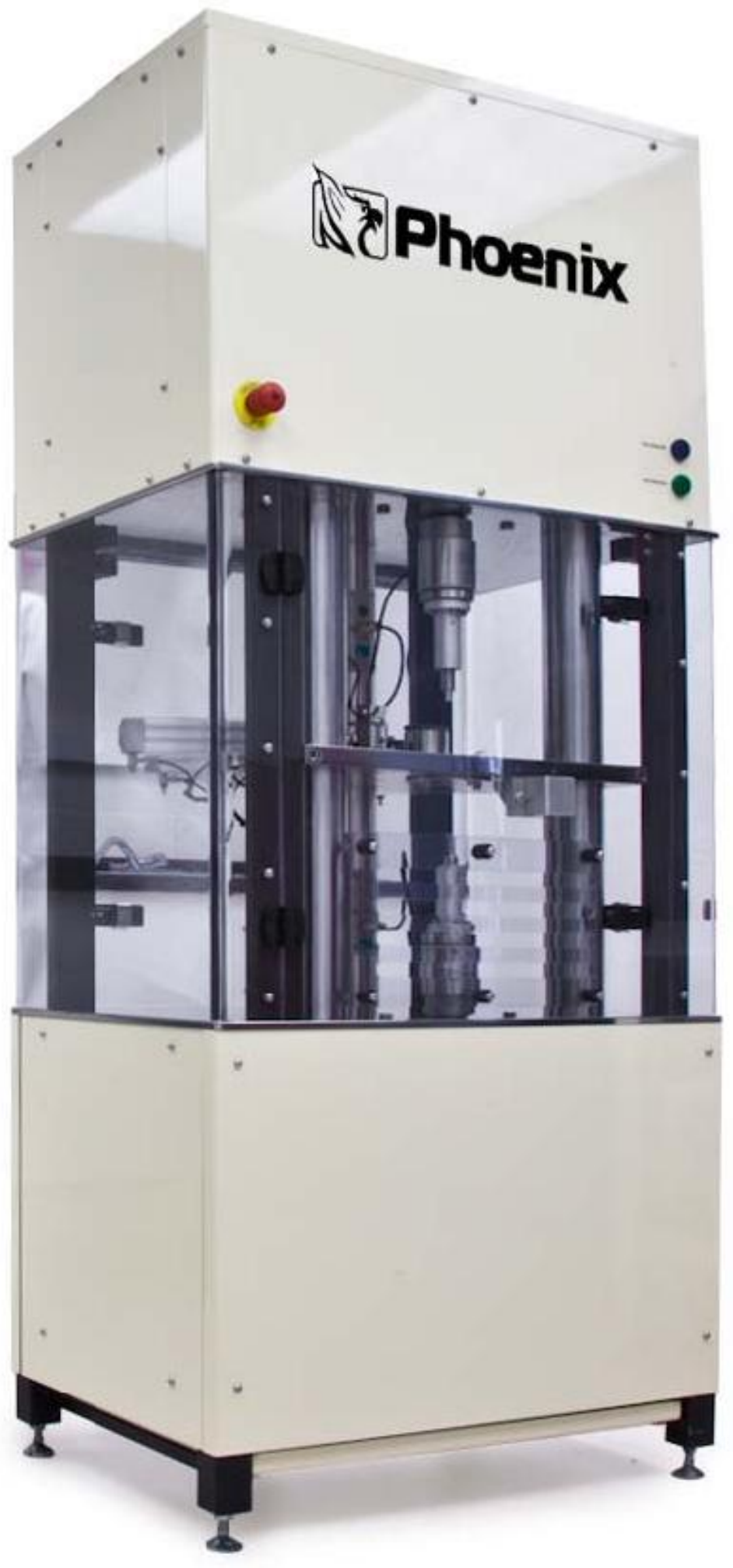




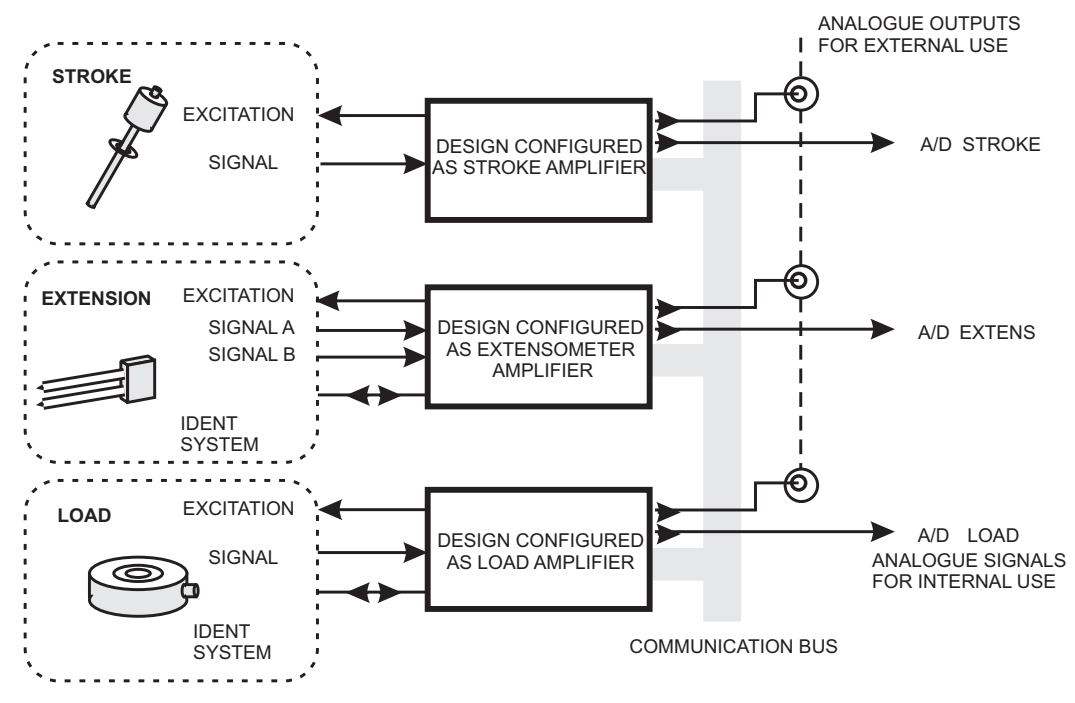




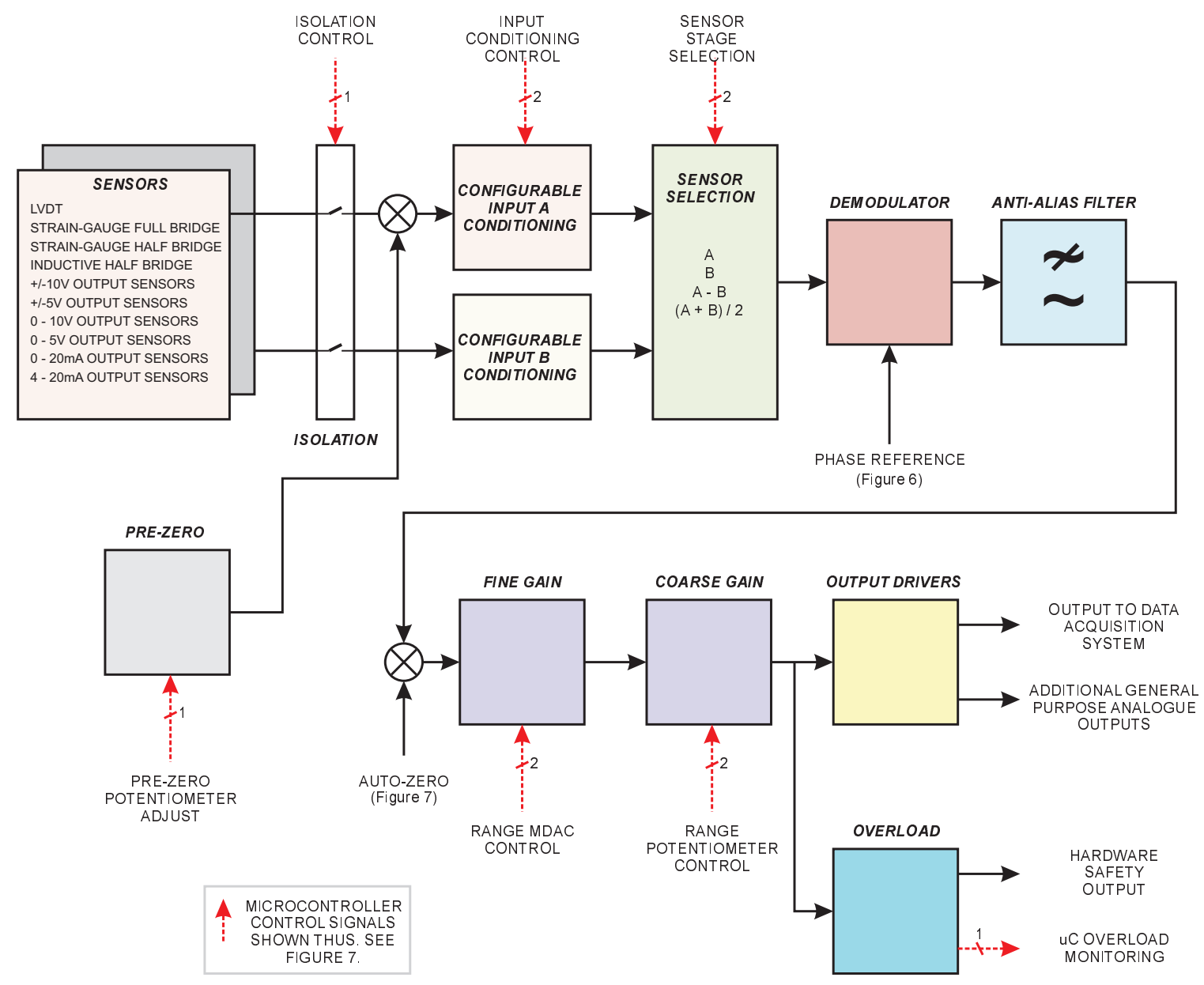




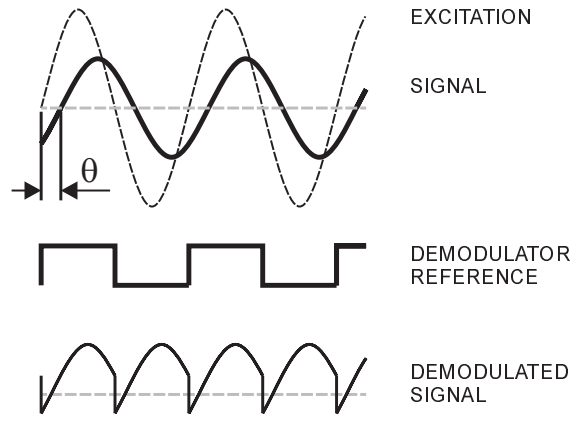




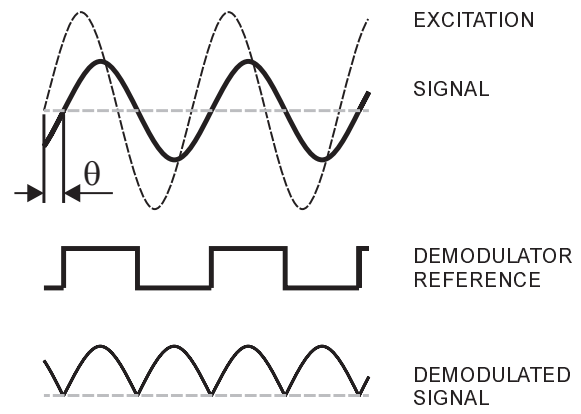




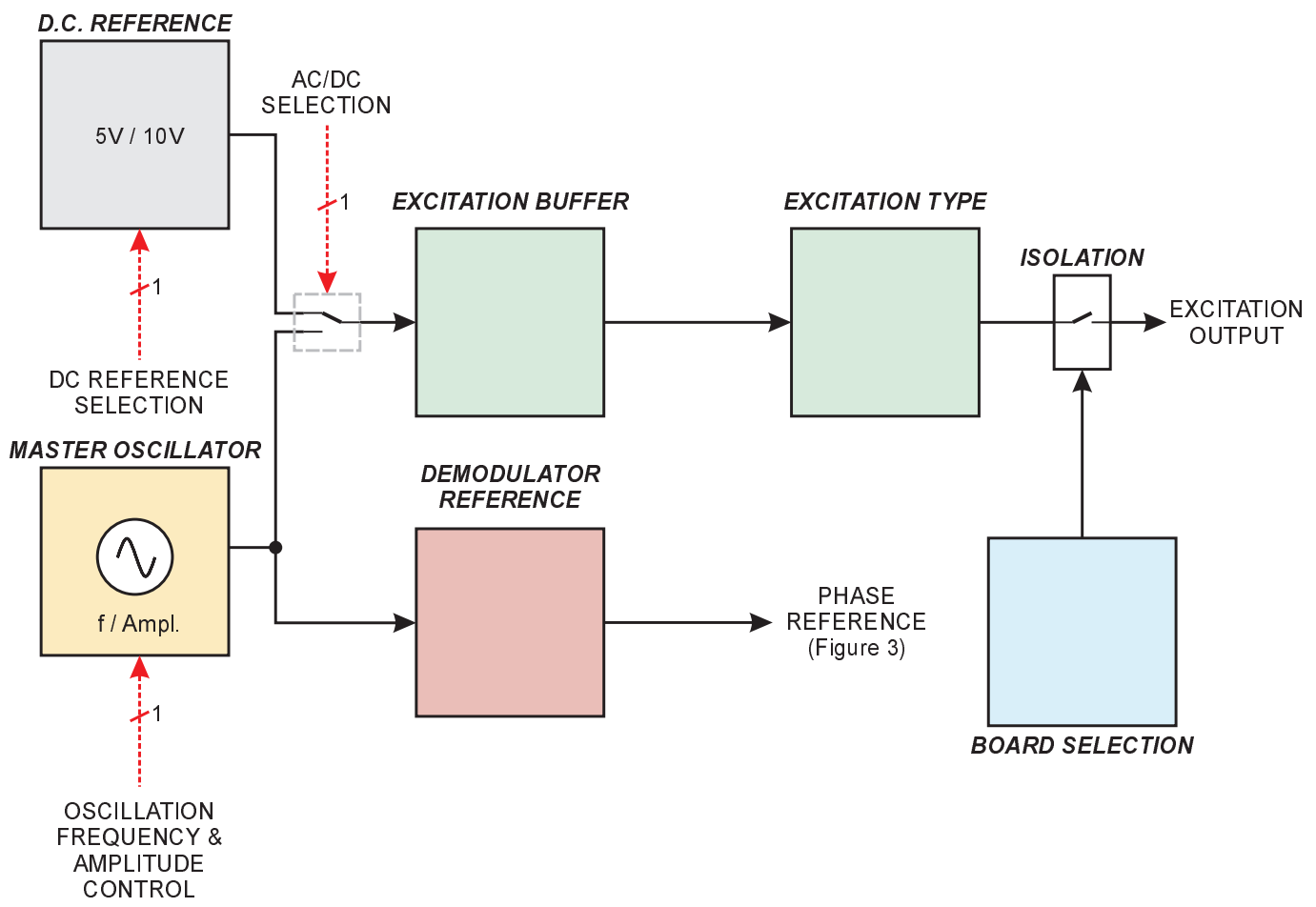




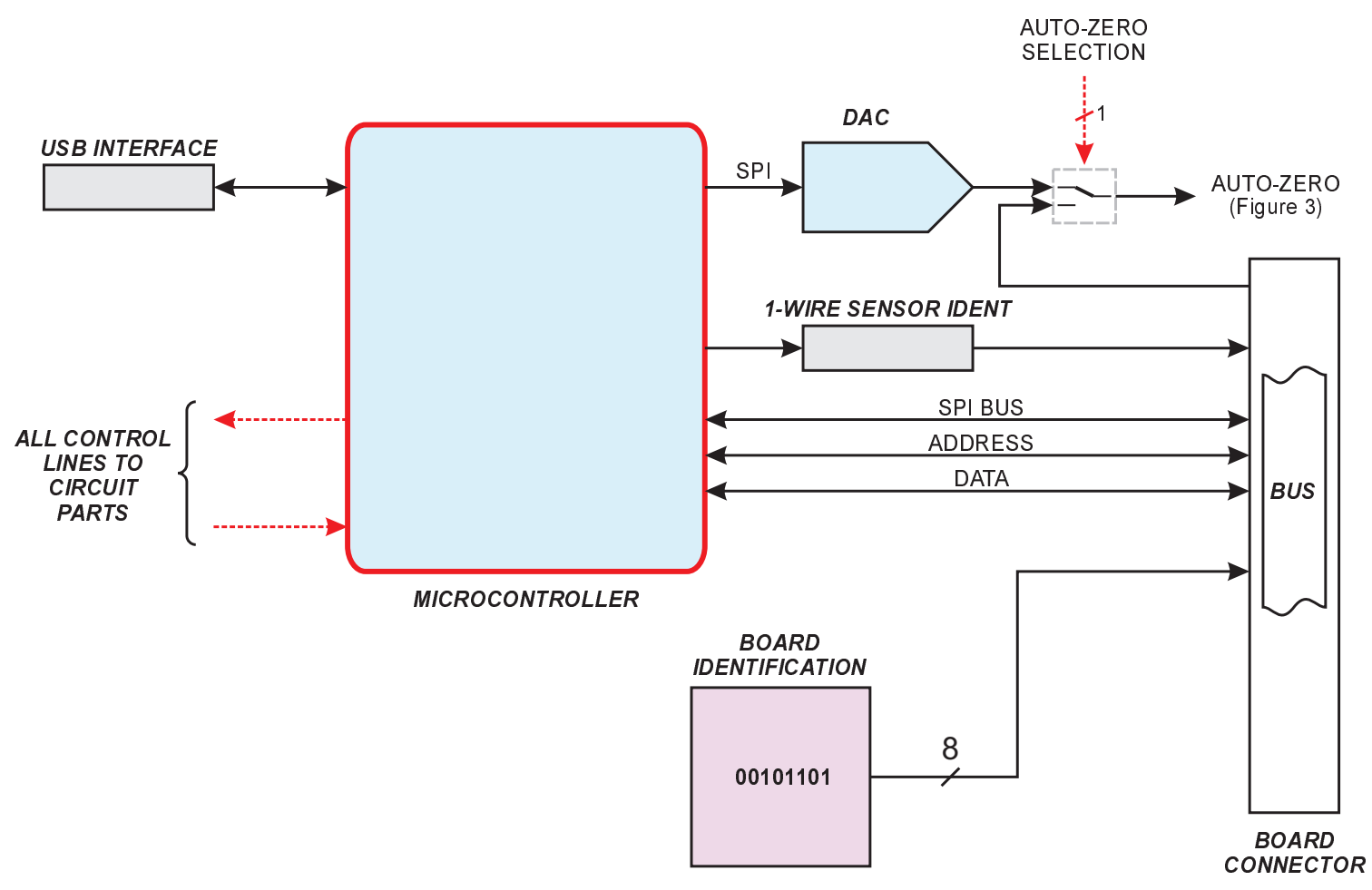




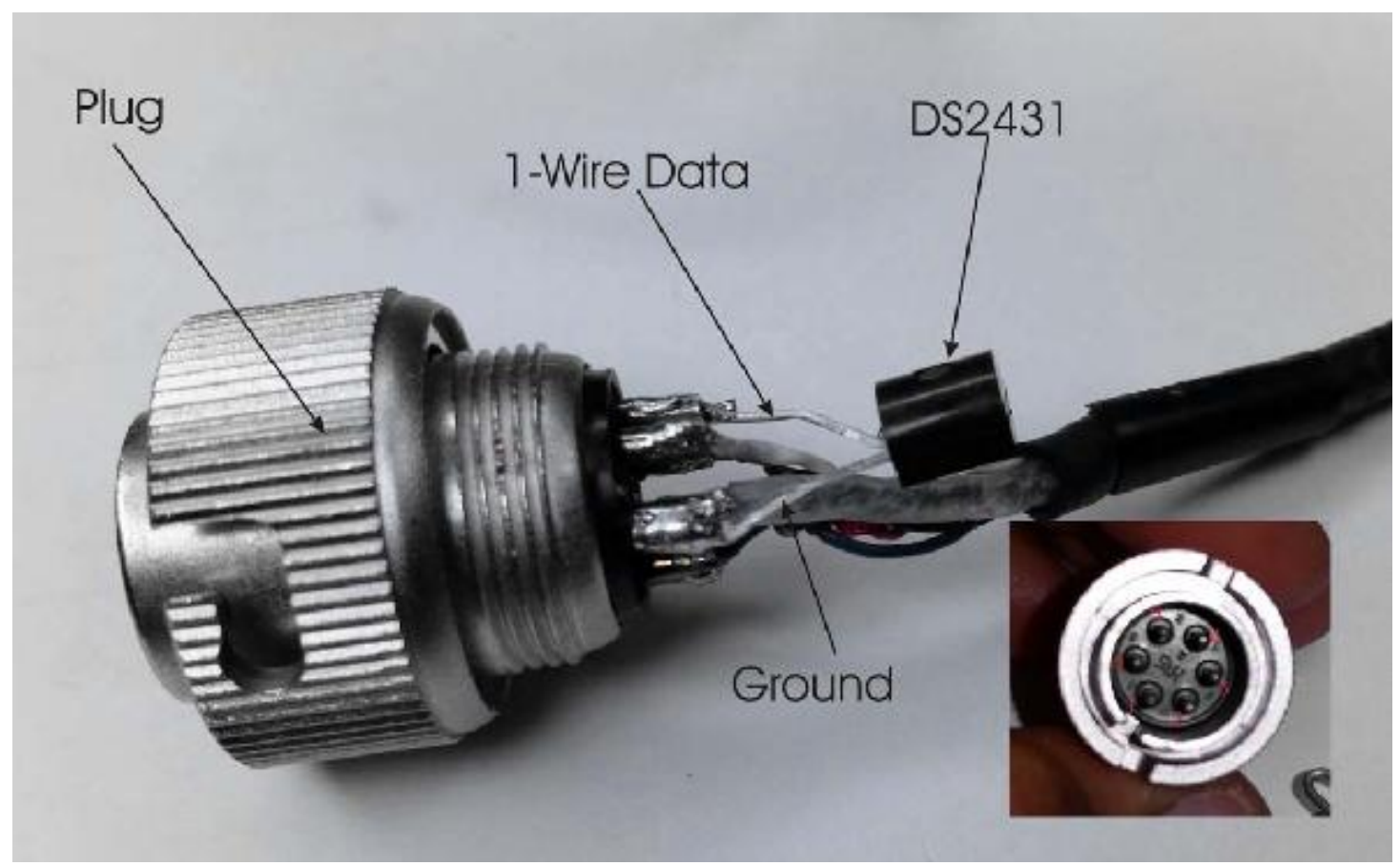




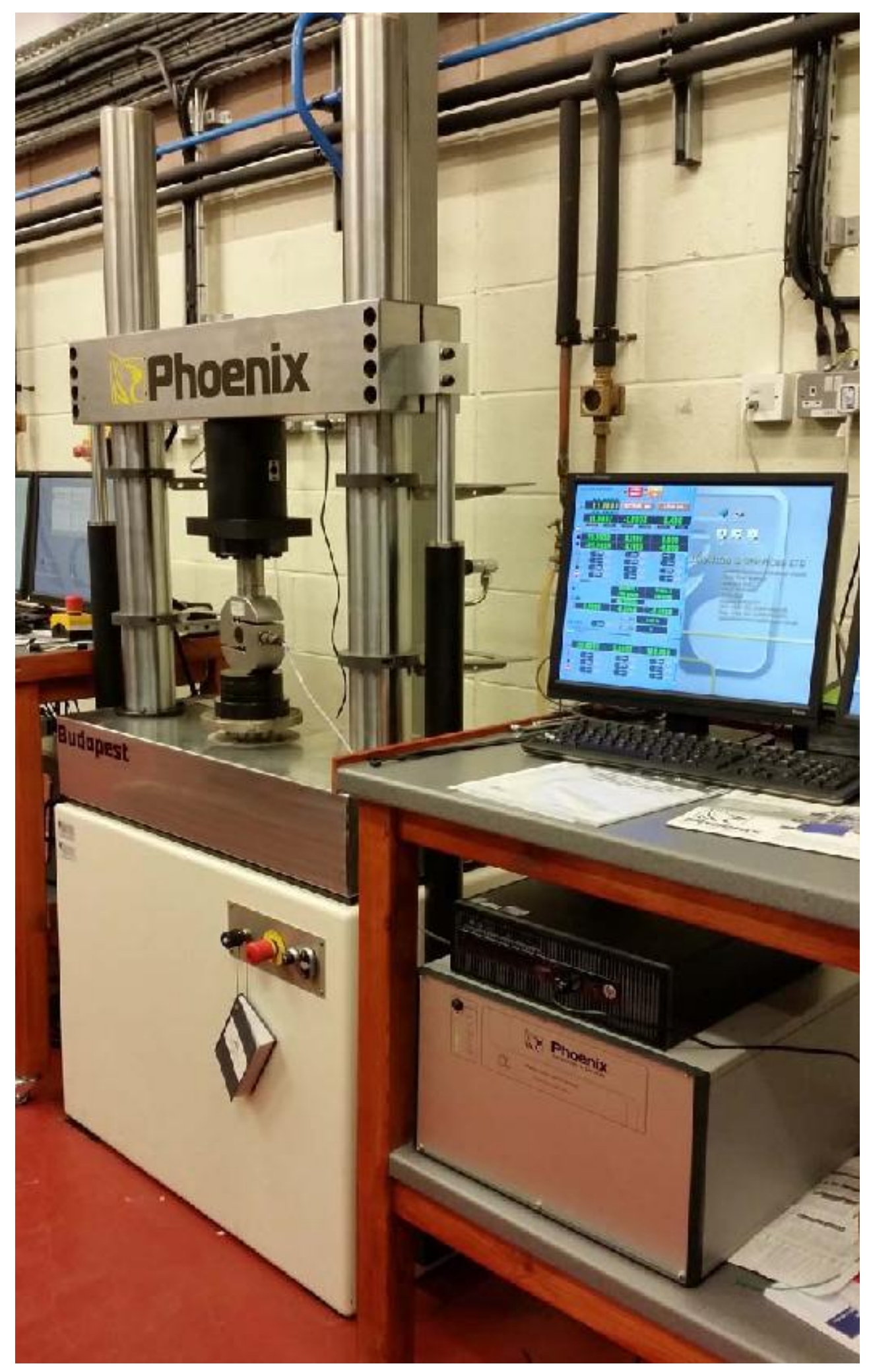




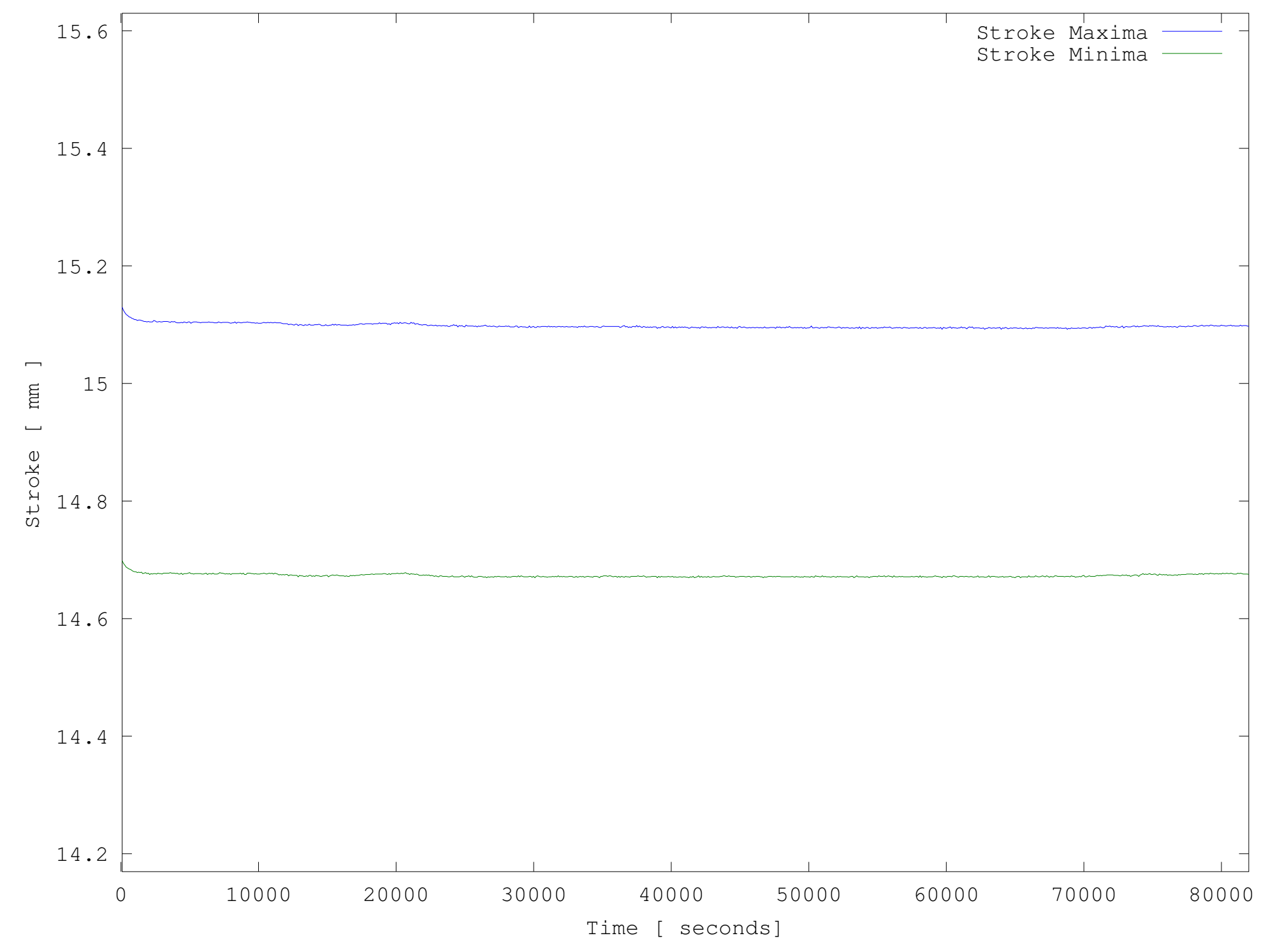




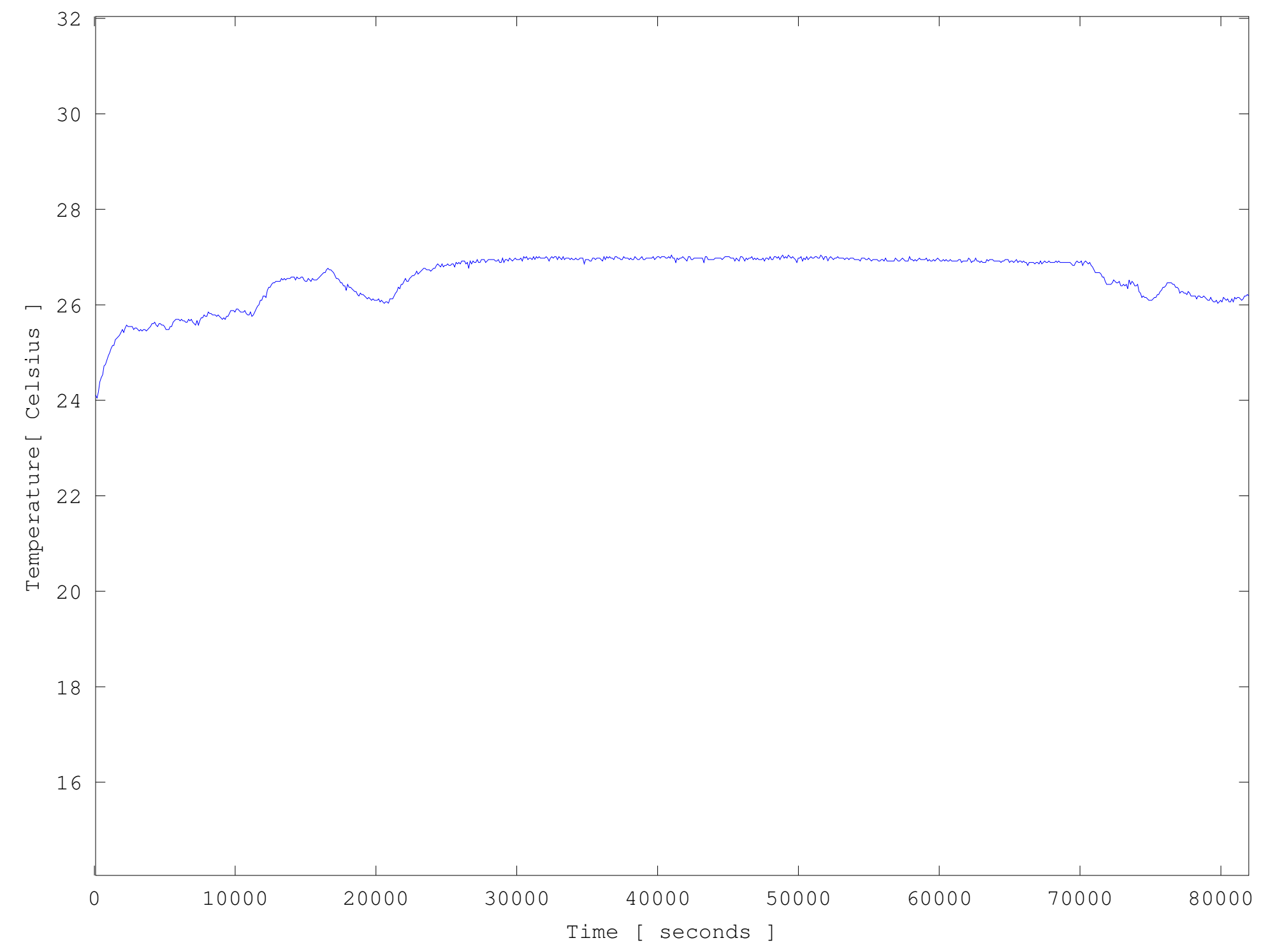




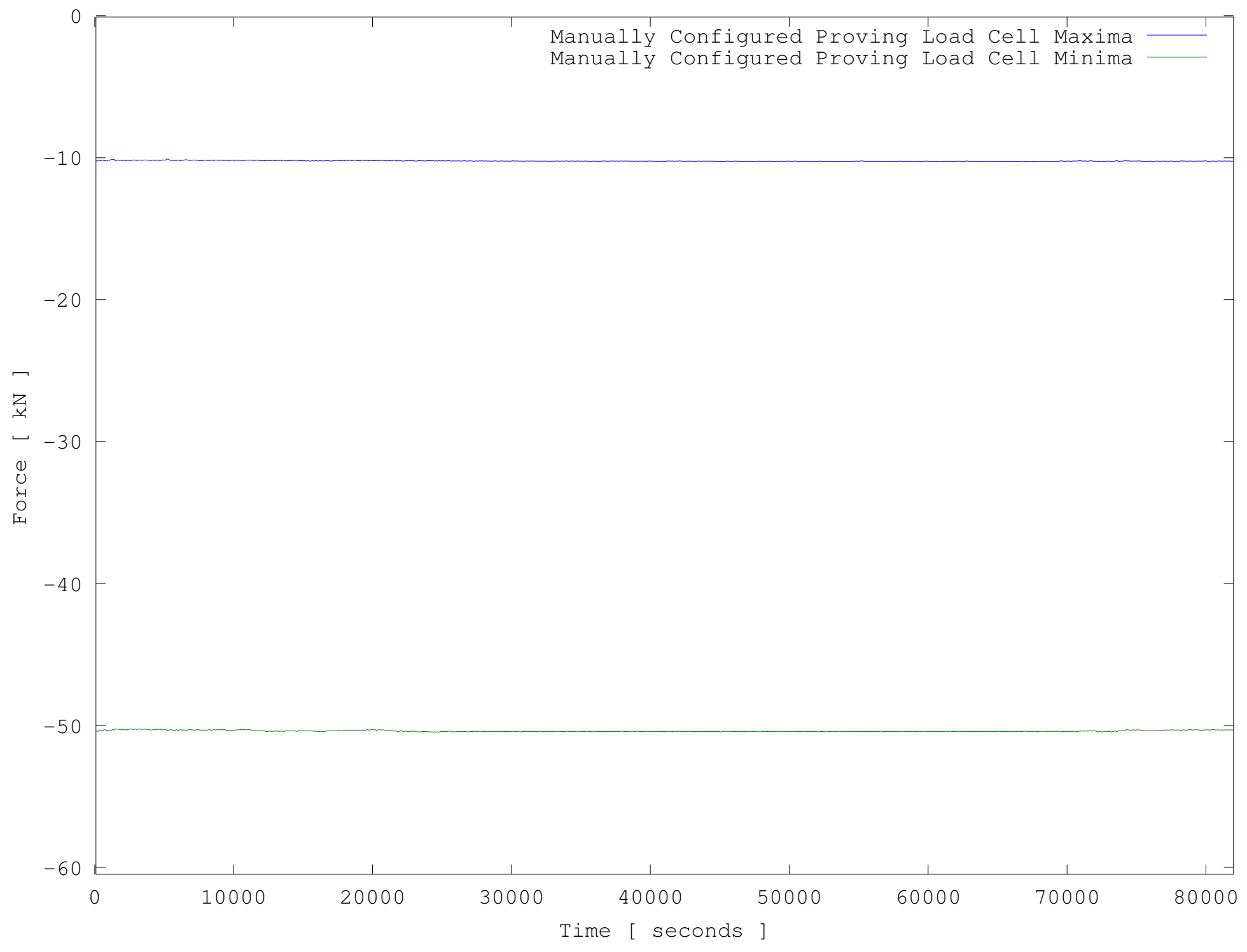




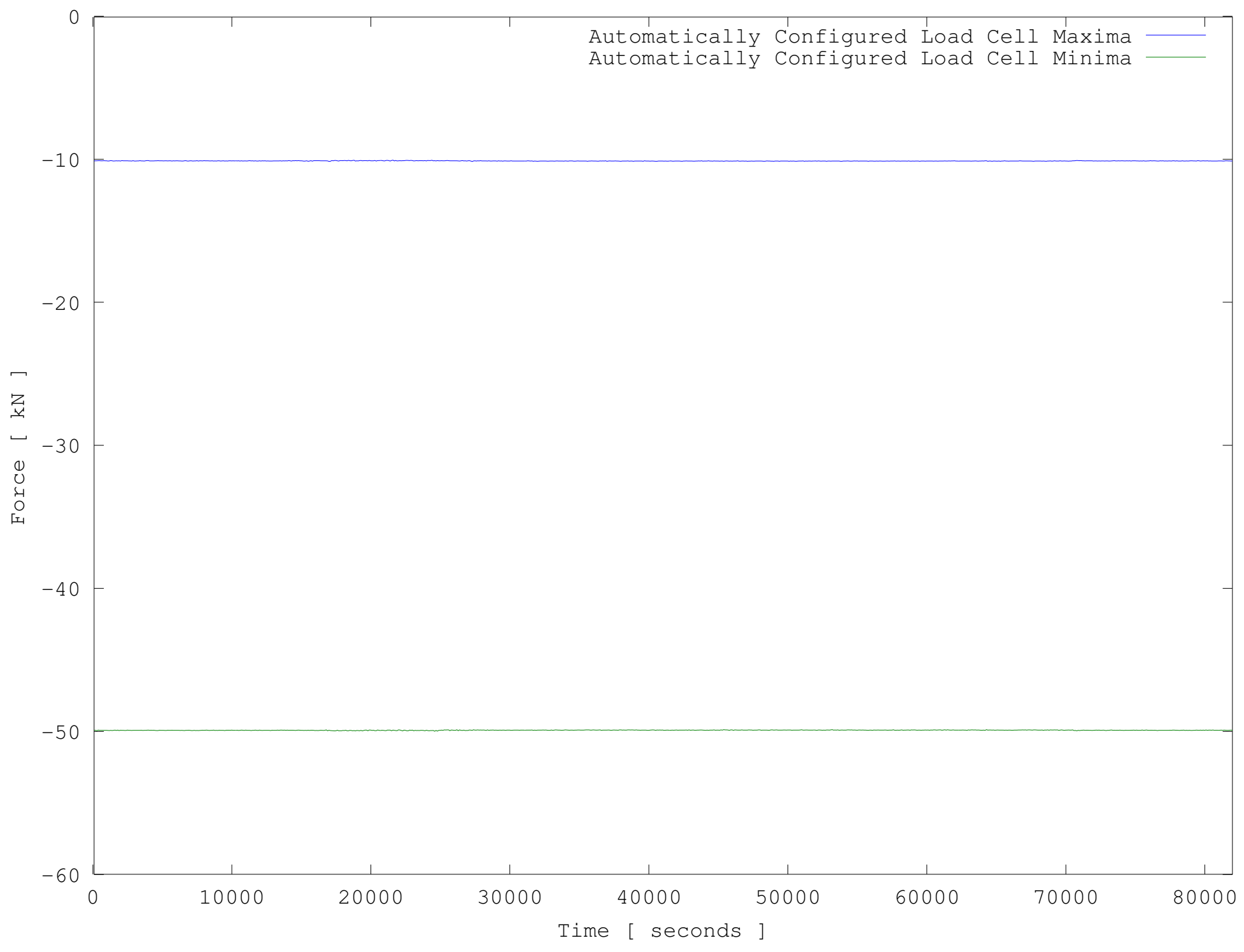


-A flexible multi-transducer signal conditioning design is proposed.

-The design is able to automatically recognise, configure and balance a wide range of industrial transducers and sensors

-Extended performance tests also show improvements in thermal drift and signal to noise ratio 
File(s) excluded from PDF

The following file(s) will not be converted:

covering letter.txt

Please click 'Download zip file' to download the most recent files related to this submission. 